\title{
Scenario Analysis on Future Internet
}

\author{
Tapio Levä, Heikki Hämmäinen, Kalevi Kilkki \\ Department of Communications and Networking \\ TKK Helsinki University of Technology \\ P.O. Box 3000, FIN-02015 TKK, Finland \\ \{tapio.leva, heikki.hammainen, kalevi.kilkki\}@tkk.fi
}

\begin{abstract}
The Internet has grown out of its original scope and scale while its importance for the society has increased. This has raised considerable uncertainties and concerns about the future evolution. We apply Schoemaker's scenario planning method to identify and analyze the key trends and uncertainties and to create four alternative evolution scenarios for the Internet. The scenarios reveal that the challenges the Internet is facing can be solved in various ways, yet leading to different network and business architectures. The results are valuable in directing research efforts to the most relevant issues.
\end{abstract}

Keywords-future; Internet; scenario analysis; network architecture; value distribution

\section{INTRODUCTION}

The importance of the Internet for the society is constantly increasing. In four decades the Internet has grown from a network of computer science researchers to a global backbone of the information society, and currently over one billion people use it to communicate, search and share information, conduct business and enjoy entertainment.

As our study regarding historical milestones of the Internet [1] shows the Internet community lead by IETF (Internet Engineering Task Force) has been remarkably successful in solving scalability bottlenecks caused by the surprising growth in user base and by novel application requirements. Nevertheless, the Internet and its architectural principles [2] were designed in the 1970s mostly for purposes that resemble very little the current and foreseen usage scenarios. These usages are questioning the ability of the Internet to cope with the forthcoming challenges.

The increased awareness of the challenges the Internet is confronting as well as of the possibilities it is offering has activated decision-makers and researchers around the world. Various initiatives have been established to study the future Internet for example in Europe (Future Internet Assembly, FIA [3]), US (GENI [4], FIND [5]), Japan (AKARI [6]), and South Korea (FIF [7]). These research efforts are seen strategically important as can be understood from the statement of Commissioner Viviane Reding that Europe must be a key player in the future of the Internet [8]. Consequently the financial investments are high; for instance European Union member states have committed over €9.1 billion of funding for ICT research [9].

Investing in the future Internet contains high level of risks because the researchers are missing the crystal ball that would tell how the funding should be used. Although the technical shortcomings could be recognized it may not be enough since the Internet evolution is affected also by economic, political and social forces that need to be understood so that technical solutions are accepted and adopted successfully. Additionally, the inertia of the Internet caused by its sheer size needs to be taken into account when planning the deployment of new technical solutions.

Creating scenarios is one way to deal with the complex uncertainties related to the Internet ecosystem. This paper describes alternative technological scenarios for the Internet over ten years (2009-2018). The scenarios are based on key trends and uncertainties identified in the frame of the Finnish Future Internet program [10]. The remainder of this paper is organized as follows. Section II introduces the scenario planning method. The process of creating scenarios is presented in the third section including the key trends and uncertainties for the future Internet. Sections IV and V describe the derived scenarios first one by one and then compared with each other. Finally, Section VI concludes the findings.

\section{SCENARIO PLANNING METHOD}

Scenario planning is an established tool for exploring complex situations with high uncertainty. Modern scenario techniques stem from war game simulations at the Rand Corporation in the 1950s [11]. In the 1970s they were used successfully in the petrochemical industry [12]. Although practitioners have developed scenario planning to several directions during the past decades (see comparison in [13]), they all build on identifying driving forces consisting of both predetermined and uncertain elements.

More recently, scenario planning has been used in dealing with high uncertainty of emerging technologies in the ICT field. Karlson et al. [14] took a holistic view and created four possible scenarios for the evolution of wireless industry from 2003 to 2015. Nordlund et al. [15] used Karlson et al.'s method to create scenarios for digital home management. Heikkinen has used scenarios to understand the usage of mobile peer-to-peer services [16, 17]. Additionally Smura and Sorri [18] have studied the wireless local area access market concentrating on indoor access and especially on rivalry between WLANs (wireless local area networks) and femtocells.

From many alternatives, Schoemaker's method [11, 1921] was seen as the most suitable one for this study. The ten- 
step framework presented in [19] was used but the last two steps requiring quantitative modeling were not carried out. To summarize the method: after definition of the time frame and the scope the key trends and uncertainties are identified. Then four internally consistent and plausible scenarios are constructed based on the most important uncertain elements. The resulting scenarios are not treated as forecasts but rather as means for bounding and understanding future uncertainties.

\section{SCENARIO CONSTRUCTION PROCESS}

In this section the process used in constructing the scenarios is presented. The findings are expressed in form of key trends and uncertainties which summarize the acquired understanding of the future Internet.

\section{A. Research Methods}

The input for the study was gathered in brainstorming sessions and interviews conducted among Finnish experts. The more detailed description of the brainstorming and interview process is presented in detail in [1].

\section{1) Brainstorming}

Key trends and uncertainties were identified in three brainstorming sessions organized in the autumn of 2008. Each session had 6-8 Finnish academics/industry experts representing different stakeholders. To cover all the important macro-environmental factors affecting the future Internet, the analysis was divided into political, economic, social, and technological domains, known also as the PEST framework.

\section{2) Expert Interviews}

Due to the rapid nature of brainstorming it was not possible to get a comprehensive understanding of identified forces, especially of uncertainties, during the sessions. Thus in addition 11 experts were interviewed in December 2008 and January 2009 in Helsinki metropolitan area. The experts represented different stakeholders including mobile device vendor, network infra vendor, and regulator. Also couple of academics specialized in networking technology, wireless networking, and network economics were interviewed.

\section{B. Key Trends}

Key trends are important factors that are certain or very likely to realize and have significant impact on the future Internet. They are underlying all four scenarios and are assumed to be valid with a reasonable probability for the chosen ten-year period. Final key trends were combined from several trends identified in the brainstorming sessions. The presentation is divided into four categories based on the PEST framework.

\section{1) Political/Regulatory Trends}

The society will be increasingly dependent on the Internet. Economy, administration, education and industry move their operations increasingly to the Internet and manual fall-backs in problem situations are disappearing. This raises governmental interest in regulative control and re-regulation.

The world (and the Internet) is moving from unipolar to multipolar. The U.S.-centered western world loses its dominant role since the rise of China, India, and other developing nations scatters the power around the globe. Additionally, the next two billion Internet users come mostly from the third world and developing nations.

The usage and allocation of spectrum will be more market-based. Increasing mobile Internet usage channels more spectrum for Internet access. Spectrum usage will be more effective and spectrum auctions are used in most countries.

Environment and energy will be more important. Environmental awareness increases and energy consumption is controlled and regulated stricter.

\section{2) Economic/Business trends (1/4)}

The world is moving from products to services. The money is on the services because producing goods is highly competed on the global space. The Internet speeds up this development.

Using ICT becomes low-cost compared to manual alternatives. Cost reductions and possibility for rationalization of business processes drive adoption of ICT in every field of economy.

Power consumption becomes a cost driver in ICT. Awareness of ICT's environmental effects increases at the same pace with improving performance and power consumption of devices. Therefore, energy efficiency becomes an important design criterion. "Green ICT" is also seen as having marketing value.

Globalization continues. This old trend continues to hold true since countries depend more and more on each other and borders disappear. However, in the future globalization will be stronger in service and knowledge industries than in manufacturing industries.

\section{3) Social Trends}

The Internet is integrating deeper into everyday life. Mapping between the real and virtual worlds tightens and people are increasingly able and willing to use Internet services. Tighter integration creates need for improvements in security, trust, and privacy.

Desire for all around availability increases. People are used to being reachable all the time with their mobile phones and now the same level of accessibility to email, social networking sites, and instant messaging is generating a demand for mobile data services. This is supported by the increasing use of location and context information.

Social networking will be faster and stronger. Social networking services gain importance and affect how people communicate and consume. For example, the increasing usage of ratings and suggestions from other consumers changes buying behavior.

Content creation will be more user-driven. The easiness of creating and sharing content in the Internet drives to YouTube and Wikipedia style of services where users are active participants and not just passive consumers.

\section{4) Technological trends}

Mobile always-on Internet connectivity increases. The Internet will be used more and more with small, portable devices like mobile phones, PDAs, and ultra-portable PCs. 
Additionally, for many new users mobile connectivity will be the first and only access method.

Performance continues to improve. Processing power improves, optical transmission boosts transfer rates and storage capacity increases. These improvements can also be seen in better price-performance ratios.

Complexity of software, services and architectures increases. Patch-on-patch tradition and new requirements increase the complexity of networks. At the same time usage of new applications is still too complex for most users. This raises usability and reliability questions to a new level.

Diversity of networks and devices increases. The Internet of things spreads ubiquitous computing quietly and increases the amount of hosts significantly. The diverse device base is connected to the Internet with a variety of access technologies. Furthermore, machine-to-machine communication brings new requirements for networking.

\section{Key Uncertainties}

Key uncertainties are important factors with uncertain direction and impact on the future Internet. While key trends form a stable ground for scenarios, key uncertainties make them distinct from each other. Key uncertainties are divided into two groups: the most important form the scenario matrix; the less important add flavor to the scenarios. Additionally, the uncertainty related to the possible collapse of the Internet is discussed separately from the scenarios. All the key uncertainties are listed in Table I and introduced indepth in the next sections.

\section{1) Network Structure}

The future Internet may either remain one whole network or it may fragment into many networks. The characteristics of these two extremes - one network vs. fragmented network - are presented by relating questions listed below.

- Will there be free connectivity in the Internet?

- Will the Internet be able to scale up?

- Will the Internet be suited to all purposes of use?

Although the Internet consists of many different networks they still form one Internet where, at least theoretically, every host is able to connect to every other host only by knowing their IP addresses. The flexibility of the Internet protocol suite has allowed an all-IP trend meaning that the IP technology is used for various networking needs including telephone and video services. This development underlines the possible cost savings that the economies of scale enable when only one network infrastructure is used. Regardless of use of the same network technology telephone traffic is today still separated from data traffic to its own network. In a truly single network there is not that kind of separation. All the traffic flows in the same wires and diverse requirements of different traffic types can be taken into account at the network level. Fundamental prerequisite of one network to be possible can be expressed as a slogan "one size fits all".

Fragmentation would mean that free end-to-end connectivity would be questioned. Extensive usage of NATs, firewalls and other middle-boxes alike disturb already
TABLE I. KEY UNCERTAINTIES

\begin{tabular}{|l|}
\hline \multicolumn{1}{|c|}{ Most Important Key Uncertainties } \\
\hline What will be the network structure? \\
\hline Ohat is the level of openness of content, applications, and hosts? \\
\hline \multicolumn{1}{|c|}{ Other Uncertainties } \\
\hline Where will the intelligence be located? \\
\hline What will be the dominating business model in the Internet economy? \\
\hline How will solutions for trust, security and authentication be implemented? \\
\hline Will the traffic be treated neutral? \\
\hline The amount of standardization: standards vs. proprietary solutions? \\
\hline Where will the standardization happen? \\
\hline \\
\hline Separate Key Uncertainty \\
\hline
\end{tabular}

nowadays end-to-end connectivity. Due to the importance of connectivity complete separation of networks does not seem feasible but the connectivity may be heavily restricted so that all the traffic between networks travels through gateways. The fragmentation does not need to happen in the physical level but it can as well - or even more probably - happen in the service level through overlay networks. These overlays borrow only the connectivity from the Internet and use their own, possibly proprietary protocols to fulfill requirements that the core Internet architecture is not capable to satisfy. These solutions, however, break the Internet architecture intentionally and thus increase the complexity of the Internet ecosystem.

Scalability (from a technical viewpoint meaning a large enough address space, fast enough routing protocols and algorithms, and small enough energy consumption) is one issue that can be solved either in the level of the Internet architecture or by building separate networks. The applicability of the Internet to every imaginable and nonimaginable purpose of use is another type of scalability issue that affects substantially the level of fragmentation. For instance, end-to-end multicasting and end-to-end quality of service cannot be supported well by the best effort type of service. Increasing real-time (video) traffic is one of those applications that have brought demand for specialized network fragments called content delivery networks (CDNs).

2) Openness of Applications, Services and Hosts

While the other scenario axis has basis in the network layer this one relates to the upper layers of the protocol stack. Applications, services and hosts may either be open like PC's and their open source software or closed like Apple's iPhone with proprietary software. These both worlds - open and closed - can be explained by relating questions listed below.

- Are the hosts freely programmable?

- Are users willing to be dependent on a single actor?

- Do users prefer bundling or buying separately? 
The world of open applications, services and hosts is the world of PC-like multipurpose devices. A single device is used to access various kinds of applications and services and is able to suffice most purposes of use. Successful and open standardization, particularly in the application level, and high availability of open source software mean that everyone has in principle the possibility to program own applications. Closed applications, services and hosts, for one, are optimized for some usages (or even for a single use). Specialization may enable better usability and fewer bugs, since all the use cases are predictable, but it restricts versatility. Security is another issue that is much easier to take into account in closed systems. Actually, Zittrain [22] sees security nuisances of open or as he expresses, generative systems, one of the most important drivers for the closed world.

All the causal factors relate to the question: who has the control over users' actions? In the open world user is the king of the hill. The Internet offers wide selection of services and user has the freedom of choice. He can install whichever applications he wants and is not locked in to some service for a long period of time. Thus the open world is naturally competitive and business model -wise mostly advertisementbased. Respectively, in the closed world, user has handed the control to some actor. Providers can most easily acquire this kind of control position through end user devices that cannot be changed to new ones as often as applications and services. There are already plenty of examples of this kind of closed devices. For instance, Microsoft's xBox, like all the other game consoles, is actually a PC performance-wise, but Microsoft decides which games are allowed to be run in it. The strong control over users creates opportunity for other business models than ad-based, e.g., subscription-based, to succeed.

User's strong position in the open world has, however, a flip side - responsibility. Openness requires more purchase decisions, and user's knowledge on purchase situations needs to be higher. Also finding and installing services rely on user's competence and activity, and the same applies to security. Bundling devices, applications, services and even networks together is one method through which a stakeholder may try to get customer locked in and dependent on a single actor. From user perspective bundling reduces the amount of purchase decisions and may thereby be an easier choice especially for technology non-enthusiasts.

3) Collapse

A larger collapse is defined here as an incident that would black-out parts of the Internet for a short period of time, cause severe economic losses and reduce people's trust on the Internet. In this respect it has significant analogies with the current financial crisis. A collapse is seen as a possibly disruptive event that wakes up the Internet community, especially decision-makers, and thus disrupts Internet's evolutional development by speeding up the implementation of new technical or regulatory means and by increasing regulative control. Fear for collapse drives for pre-emptive actions like improving the resiliency of networks and services.
Depending on its nature, a larger collapse could create pressure to whichever direction following both scenario axes (although pressure towards network fragmentation and closed applications, services and hosts seems stronger) meaning that a larger collapse will rather lead to some scenario, not vice versa. Hence the collapse uncertainty is studied separately from the scenarios by identifying several possible causes and their consequences in [1].

\section{4) Other Key Uncertainties}

The other key uncertainties with their extreme outcomes are presented briefly in this section. Inspired by Karlson et al. [14], we have created a five-level scale between the two extremes and assigned feasible values for each scenario (Figure 1).

\section{a) Where will the intelligence be located?}

Originally the Internet was a dumb network connecting smart hosts. The hosts were equal in their capabilities and roles. Client-server model used widely in the Web differentiated the roles of the hosts. High level of intelligence in clients indicates more important role of peer-to-peer model, whereas significant amount of intelligence in servers speaks for client-server model. It is also constantly questioned if any intelligence should be inserted to the network. Thus the question here is divided into two questions: 1) client vs. server and 2) end points vs. network.

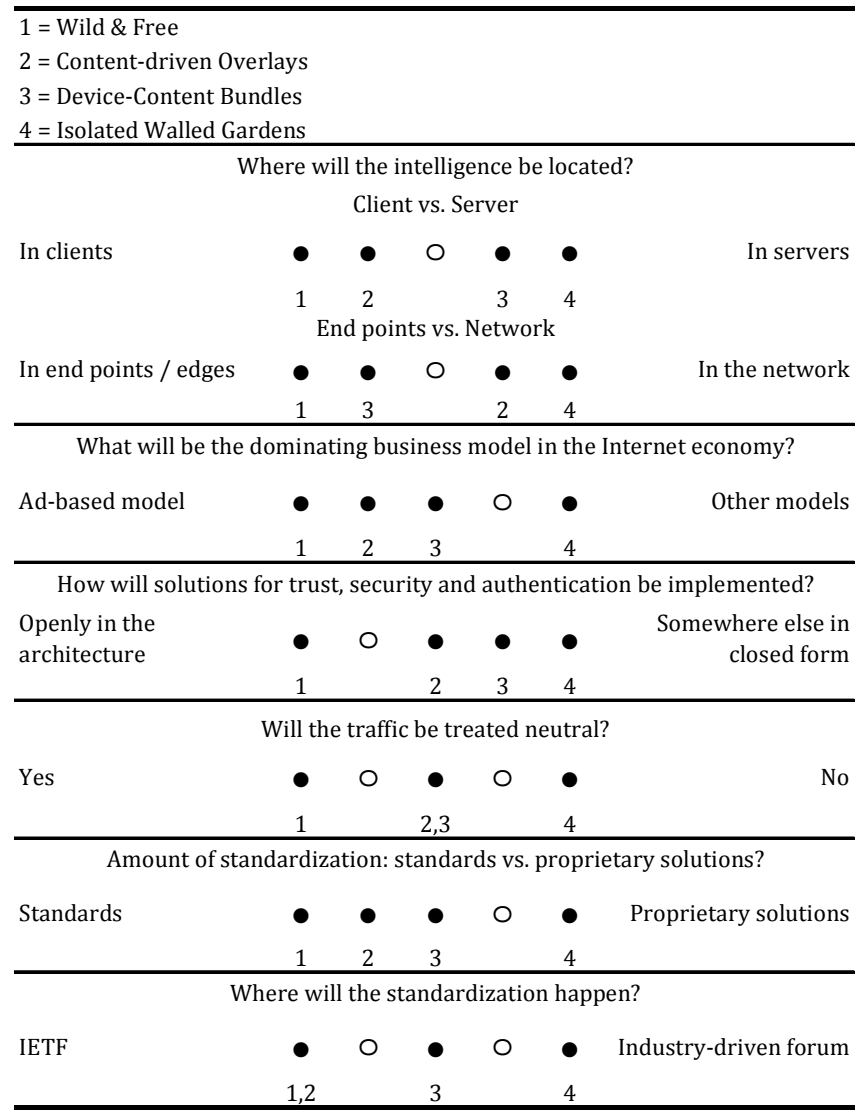

Figure 1. Other key uncertainties valued in five-level scale 
b) What will be the dominating business model in the Internet economy?

Simple and "free" ad-based business model has been by far the most successful revenue model when Internet services are considered. Transaction-based business model, like paying with PayPal or credit cards, has been mostly used when physical goods are sold through the Internet. Additionally, subscription-based model would be highly interesting to companies and simple enough for users. Thus the big question here is, will the Internet business be mostly ad-based or do other models break through?

c) How will solutions for trust, security and authentication be implemented?

Lack of trust, security and authentication is a recognized challenge that needs to be tackled somehow, at least in the case of mission-critical applications. Universal, open solutions built in the architecture are a reasonable option, but closed solutions relating for example to separate network or provider-controlled solutions in closed architecture are other choices.

\section{d) Will the traffic be treated neutral?}

Principle of net neutrality requires that all content, sites, and platforms are treated equally [23]. In a neutral network traffic flows related to for instance e-banking, video streaming, peer-to-peer file sharing or emailing are not treated differently but they all have same priority level from the network perspective. Blocking content and communication is one of the things that violate net neutrality.

e) Amount of standardization: standards vs. proprietary solutions?

The Internet architecture relies heavily on open standards called RFCs (Request for Comments). On the application and service level, proprietary solutions have, however, an important role. For example, some important network overlays, VoIP network Skype and peer-to-peer file sharing network BitTorrent, are based on proprietary solutions. Standards allow competition, while proprietary solutions enable emergence of monopolistic pockets. The question here is: will the Internet be based mostly on standards or on proprietary solutions?

\section{f) Where will the standardization happen?}

Internet-related issues have been traditionally standardized in the IETF. After the commercialization, other forums have emerged including W3C (The World Wide Web Consortium) concentrating on Web standards and 3GPP (The Third Generation Partnership Project) working for the third generation mobile phone system. Standardization could also be done in the industry-driven forums that would be open only for part of the Internet industry.

\section{SCENARIOS}

The final scenarios were formed iteratively based on the interviews. The two most important key uncertainties were crossed to form a scenario matrix. Finally the scenarios were named descriptively to illustrate their idea immediately. The scenario matrix including some descriptive characteristics of each scenario is presented below in Figure 2. More accurate descriptions are available in Sections A-D.

\section{A. Wild \& Free}

In the wild and free Internet a multitude of services and applications are offered in a single network. Users have versatile, freely programmable devices that can connect freely to each other and to any available content, service and application. Free connectivity has reached its ultimate level since access networks have been opened and all users can connect to the Internet through whichever network they want. Consumers are kings who enjoy the ever-increasing supply of new services and are not willing to constrain on the service offering of a single company. Due to the absence of lock-ins consumers can easily switch from a service to

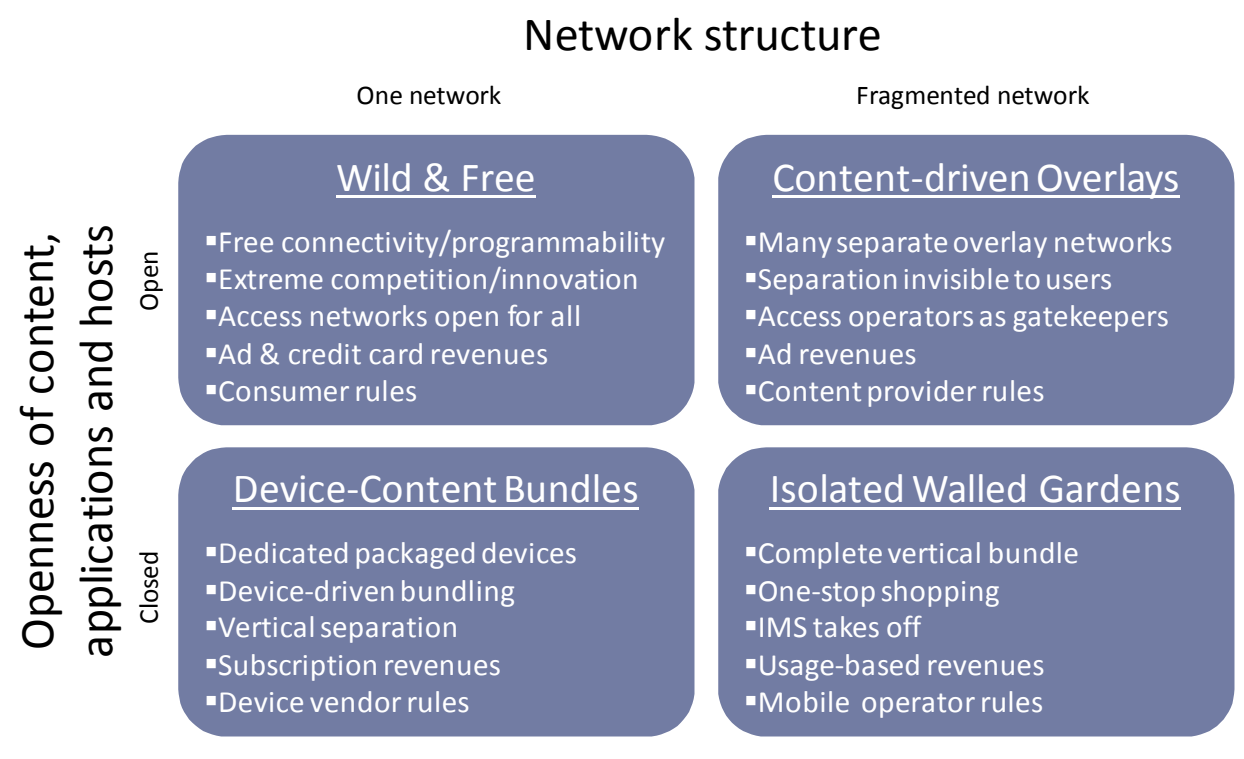

Figure 2. Scenario matrix 
another, if a better service becomes available. Thus it is difficult for other business models to compete with advertisement-based model offering the service "for free" to the customers.

The current and forthcoming scalability problems have been solved so that the Internet can handle both increasing amount of users, hosts and traffic as well as old and novel usage scenarios with diverse service level requirements. Success in solving the core problems in technological domain has allowed regulator to concentrate on enabling competition and innovation in free markets. The last monopolistic pocket in the Internet, access networks, is brought down, and thus the industry is completely horizontal and extremely competitive. The IETF has come to its own and standardization and open source software are seen important. Trust, security and authentication are implemented openly in the architecture, but only concerning those applications that require them. Due to free programmability and imbalance between regional regulations, unwanted traffic remains as a serious problem and the race between malware makers and security companies continues.

\section{B. Isolated Walled Gardens}

Isolated walled gardens are access operator centric network fragments which bundle all the components devices, network and content - together. The complete vertical bundle means that access operator has control over end users and content. Devices, software and Internet connection are sold at the same time by access operators which also take the responsibility for managing the complete package remotely. This decreases the amount of (purchase) decisions needed and makes life easy for consumers. Technology-wise Isolated Walled Gardens is the IMS (IP Multimedia Subsystem) world. Refined usage-based monthly billing is possible and is used extensively like in the telco world.

Some network fragments are interoperable, while others are isolated by design. However, interworking between competing access operators is always separately negotiated, which means more proprietary solutions and standardizing interfaces only. A strong industry forum driven by access operators has replaced IETF-style of standardization. This change combined with restricted programmability results in the situation where open innovation and entering the Internet business becomes much more difficult leading to oligopolistic markets and higher prices. Internet pioneers and academics are longing for "the good old Internet", while many users are satisfied with better security, quality of service and trust between users.

\section{Content-driven Overlays}

Because the common Internet architecture has not been able to support all the varying service requirements of different applications, the network is fragmented into overlays based on content type and application. Companies, mostly content providers, offer better quality of service in separate networks optimized for specific services, for example, video streaming. At the same time governments and companies have built secure networks for critical functions of the society. Although all these overlays are built on the top of IP, they are optimized for certain use and have much tighter control over the traffic generated by end users. To achieve better performance and conformance to standards, the common Internet architecture is intentionally broken when needed, which increases the complexity of the networks.

Building of dedicated networks is paid by competing content providers which try to attract as many customers as possible to maximize advertiser value. This puts companies in unequal position since those providers which are able to pay for better quality, get it, and those which cannot afford, must content themselves on poorer quality. This favors large players which can attract more customers and thus more advertisers by offering better services. From consumer point of view the situation is good since fragmentation of the networks is invisible to them, and they just enjoy broad variety of free, good quality services. Additionally, regulators fight for open competition in the markets, which keeps prices low, decreases companies' possibilities to get customer lock-in, and favors ad-based business model. Although content providers skim the cream off the cake access operators have important role in forwarding the traffic to the right overlay network.

If optimized network fragments fully take over, the development of the basic, best effort Internet may be stopped since the concentration is on content-driven overlays. Nevertheless, the network can also defragment, if an overlay (e.g., information networking or social networking) solves the largest problems that lead to the fragmentation.

\section{Device-Content Bundles}

While Internet usage moves increasingly from PCs to mobile phones and other portable devices, users' interest on installing applications and updating their devices themselves decreases. At the same time device vendors offer tempting device-content bundles which combine devices and services in a seamless manner. Due to their unbeatable user experience, including enhanced reliability and security, and hyper-usability, consumers are willing to accept lock-in. The Internet is still whole, but consumers choose which part of the service offering is available to them when they purchase their devices.

Updating and installing new services is possible only through device vendor controlled service portals meaning that device vendors take their share of all purchases. Restricted ability to install applications raises the role of web applications and client-server model which changes user devices from active participants to merely passive terminals and increases the importance of service clouds. This development leads to closed and more dedicated devices, resembling today's Xboxes and iPhones. Device manufacturers' control enables subscription-based revenue models in which device price includes access to content and services for some period of time. Nokia's Comes with Music handsets are an early example of this kind of innovative bundle [24]. 


\section{COMPARISON OF SCENARIOS}

In the previous section the scenarios were described one by one. In this section they are compared in terms of technical and business architecture and value distribution between different stakeholders.

\section{A. Architecture in Scenarios}

The architectural differences of the scenarios are illustrated in Figure 3. The presentation is simplified and takes into account only end content, network, and end user devices. Content covers not only textual, audio and video data but also services and applications. The architecture can be understood both as business and as technical architecture which are uniform in the scenarios.

Difference in network structure separates the left-hand side scenarios from the right-hand side scenarios. Openness of applications, services and hosts determines how devices can access the content. For example the Wild \& Free and Device-Content Bundles scenarios share the same architecture with the exception that in Device-Content Bundles the device in use defines which part of the content is accessible.

In Content-driven Overlays the content and network are bundled together but devices can connect to every network, whereas in Isolated Walled Gardens all the elements device, network and content - are bundled together. Although the walled gardens are isolated, communication between users belonging to different networks is restrictedly possible through access operator controlled gateways.

\section{B. Value Distribution in Scenarios}

The power positions of the most important stakeholders are illustrated by presenting value distribution in scenarios. Figure 4, inspired by Christensen et al. [25], depicts in a simplified manner to whose pockets the profits are flowing in. Device vendors include end-user device manufacturers like Nokia, Apple and Dell. Content providers cover software companies (Microsoft, SAP), Internet service giants (Google, Yahoo), media houses (NBC, BBC) and
Wild \& Free

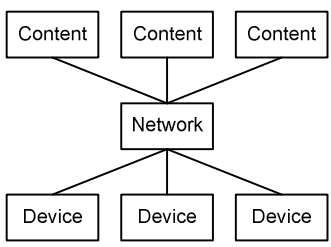

Device-Content Bundles

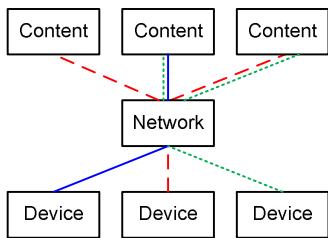

$\underline{\text { Content-driven Overlays }}$

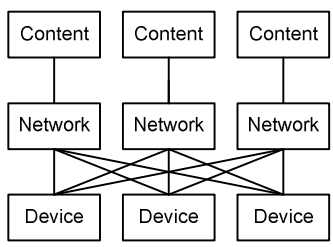

Isolated Walled Gardens

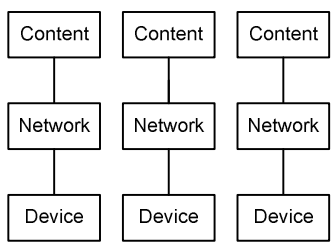

Figure 3. Simplified business and technical architecture in the scenarios

entertainment companies (Disney, Universal). Access operators include traditional ISPs like Comcast and Verizon as well as mobile operators like Vodafone and Orange. The role of backbone operators is analyzed neither here nor elsewhere in this paper since that did not come up during the research process. The reason for this may be either that the experts saw their role stable or they were not able to speak out due to the lack of large backbone operators in Finland.

The presentation is qualitative, although the money is presented as amount of coins. Thus only the ratio of coins inside a scenario and between the scenarios should be examined. For example, Device-Content Bundles is more profitable for device manufacturers than for access operators while Content-driven Overlays offers best prospects for content providers. One important issue to clarify is the difference in the amount of coins between Wild $\&$ Free and other scenarios. Due to the extreme competition covering every business sector the profits are smaller than in other scenarios since a larger part of the money remains in customers' pockets.
Wild \& Free

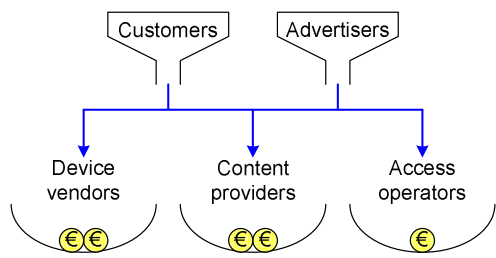

$\underline{\text { Content-driven Overlays }}$

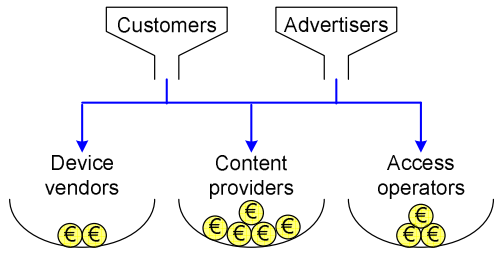

$\underline{\text { Isolated Walled Gardens }}$
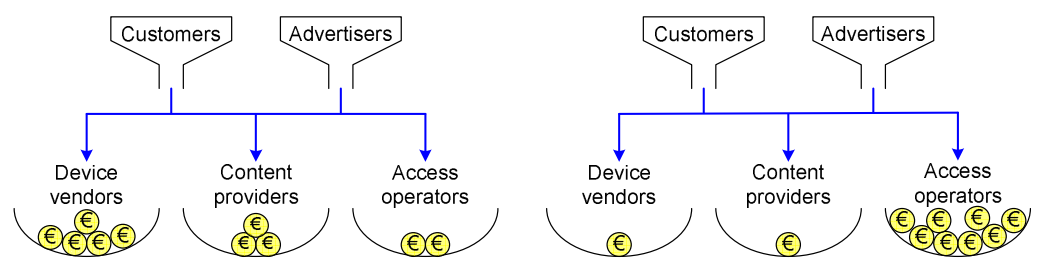

Figure 4. Simplified value distribution in the scenarios. 
Which scenario is best for the society? Regulator's goal is to maximize social welfare which is compounded of customer surplus and producer surplus [26]. Based on this examination, however, it is impossible to say which of the scenarios would be optimal from regulator's perspective.

\section{CONCLUSION}

The main purpose of scenario analysis is to clarify our thoughts on the possible future advances in certain technical or business field. A proper analysis can limit the amount of uncertainties, generate consistent pictures, and identify issues that might otherwise remain ignored. However, it should be noted that the actual outcome ten years from now will likely be a hybrid of several scenarios.

The key finding derived during the scenario process was that the challenges the Internet is encountering can be solved at many levels. As the scenario axes propose, there are three possible ways to satisfy emerging requirements. First, IETF strives for openness and network integrity preserving solutions implemented in the core Internet architecture. Second, business interests of various stakeholders and failures in implementing some features in the core architecture pave the way for solutions that rely on building separate network fragments or closing the applications, services, and hosts. Third, a completely new clean slate approach might be able to solve a bunch of problems, although the deployment conditions of a complete redesign are not studied closer in this paper.

The strength and relevance of uncertainty-based scenarios depend on the match of technical and business architectures in each distinctive scenario. Each produced scenario corresponds to a characteristic technical and business architecture, which may trigger valuable forwardlooking debates among experts. Differences between the constructed scenarios concerning the power relationships and value distribution between stakeholders reveal the underlying tensions and differing interests of stakeholders.

According to Schoemaker [21], scenario planning should stimulate decision makers to consider changes they would otherwise ignore. Thus scenarios are typically used for planning strategic actions of a given market player. We have used the produced scenarios to help draft the Internet research and standardization strategy of the Finnish Future Internet research program [1].

\section{ACKNOWLEDGMENT}

This research was conducted in the Future Internet program of Tivit (Finnish Strategic Centre for Science, Technology and Innovation in the field of ICT), funded by the Finnish Funding Agency for Technology and Innovation (Tekes), and coordinated within the Econ@Tel (COST605) network.

\section{REFERENCES}

[1] T. Levä, "Scenario Analysis on Future Internet", MSc Thesis, Helsinki University of Technology, 2009.

[2] B. Carpenter, "Architectural Principles of the Internet", IETF RFC 1958, 1996.
[3] FIA: Future Internet Assembly, web page. Available at http://www.future-internet.eu/, accessed 27 March 2009.

[4] GENI: Global Environment for Network Innovations, web page. Available at http://www.geni.net/, accessed 27 March 2009.

[5] FIND: Future Internet Design, web page. Available at http://www.nets-find.net/, accessed 27 March 2009.

[6] AKARI: Architecture Design Project for New Generation Network, web page. Available at http://akari-project.nict.go.jp/eng/index2.htm, accessed 27 March 2009.

[7] FIF: Future Internet Forum, web page. Available at http://fif.kr/, accessed 27 March 2009.

[8] V. Reding: "Internet of the future: Europe must be a key player", speech to the Lisbon Council on 2 February 2009. Available at http://ec.europa.eu/commission_barroso/reding/docs/speeches/2009/b russels-20090202.pdf, accessed 27 March 2009.

[9] European Commission, "Bled Declaration". Available at http://www.future-internet.eu/publications/bled-declaration.html, accessed 27 March 2009.

[10] ICT SHOK Future Internet Programme of Finland, web page. Available at http://futureinternet.fi/, accessed 27 March 2009.

[11] P.J.H. Schoemaker, "Multiple Scenario Development: Its Conceptual and Behavioral Foundation," Strategic Management Journal, vol. 14, pp. 193-213, 1993.

[12] P. Wack, "Scenarios: Uncharted Waters Ahead", Harvard Business Review, vol. 63, pp. 73-89, 1985.

[13] R. Bradfield, G. Wright, G. Burt, G. Cairns, and K. Van Der Heijden, "The origins and evolution of scenario techniques in long range business planning", Futures, vol. 37, pp. 795-812, 2005.

[14] B. Karlson, A. Bria, P. Lönnqvist, C. Norlin, and J. Lind, Wireless Foresight: Scenarios of the mobile world in 2015. Chichester, UK: Wiley, 2003.

[15] T. Nordlund, M. Tallberg, and H. Hämmäinen, "Scenarios for management of digital homes", Proceedings of $6^{\text {th }}$ Conference on Telecommunication Techno-Economics (CTTE 2007), pp. 1-8, 2007.

[16] M.V.J. Heikkinen and H. Hämmäinen, "Scenario Planning of Mobile Peer-to-Peer Service Usage", Proceedings of 7th International Conference on Mobile Business (ICMB 2008), pp. 145-152, 2008.

[17] M.V.J. Heikkinen, M. Matuszewski, and H. Hämmäinen, "Scenario planning for emerging mobile services decision making: mobile Peerto-Peer Session Initiation Protocol case study", Int. J. Information and Decision Sciences, vol. 1, pp. 26-43, 2008.

[18] T. Smura and A. Sorri, "Future scenarios for local area access: industry structure and access fragmentation", Proceedings of the Eighth International Conference on Mobile Business (ICMB 2009), Dalian, China, June 27-28, 2009.

[19] P.J.H. Schoemaker and M.V. Mavaddat, "Scenario Planning for Disruptive Technologies", in G.S. Day and P.J.H. Schoemaker (eds.): Wharton on Managing Emerging Technologies, New Jersey:Wiley, pp. 206-241, 2000.

[20] P.J.H. Schoemaker, "When and how to use scenario planning: A heuristic approach", Journal of Forecasting, vol. 10, pp. 549-564, 1991.

[21] P.J.H. Schoemaker, "Scenario planning: A tool for strategic thinking", Sloan Management Review, vol. 36, pp. 25-40, 1995.

[22] J. Zittrain, The Future of the Internet - And How to Stop It. New Haven \& London: Yale University Press, 2008.

[23] T. Wu, Network Neutrality FAQ, web page. Available at http://timwu.org/network_neutrality.html, accessed 27 March 2009.

[24] Nokia, Comes with Music service, web page. Available at http://www.comeswithmusic.com/, accessed 27 March 2009.

[25] C.M. Christensen, M. Raynor, and M. Verlinden, "Skate to where the money will be", Harvard Business Review, vol. 79, pp. 72-81, 2001.

[26] C. Courcoubetis, R. Weber, Pricing Communication Networks, Wiley, 2003. 